\title{
Exploring the Self-Assembly of Encapsulin Protein Nanocages from Different Structural Classes
}

\author{
India Boyton $^{+\dagger}$, Sophia C. Goodchild’, Dennis Diaz ${ }^{\dagger}$, Aaron Elbourne ${ }^{\ddagger}$, \\ Lyndsey Collins-Praino ${ }^{\dagger t} \&$ Andrew Care ${ }^{+\dagger}$ *
}

\author{
${ }^{+}$School of Life Sciences, University of Technology Sydney, NSW 2007, Australia \\ ${ }^{\dagger}$ Department of Molecular Sciences, Macquarie University, NSW 2109, Australia \\ ${ }^{\ddagger}$ School of Science, College of Science, Engineering and Health, RMIT University, \\ Melbourne, VIC 3000, Australia \\ $\$$ Adelaide Medical School, The University of Adelaide, Adelaide, 5005, SA, Australia. \\ $\dagger$ ARC Centre of Excellence for Nanoscale BioPhotonics, Macquarie University, NSW 2109, \\ Australia \\ ${ }^{\S}$ ARC Centre of Excellence in Synthetic Biology, Macquarie University, NSW 2109, \\ Australia \\ *andrew.care@uts.edu.au
}

\begin{abstract}
Encapsulins, self-assembling icosahedral protein nanocages derived from prokaryotes, represent a versatile set of tools for nanobiotechnology. However, a comprehensive understanding of the mechanisms underlying encapsulin self-assembly, disassembly, and reassembly is lacking. Here, we characterise the disassembly/reassembly properties of three encapsulin nanocages that possess different structural architectures: $T=1(24 \mathrm{~nm}), T=3$ (32 $\mathrm{nm})$, and $T=4(42 \mathrm{~nm})$. Using spectroscopic techniques and electron microscopy, encapsulin architectures were found to exhibit varying sensitivities to the denaturant guanidine hydrochloride $(\mathrm{GuHCl})$, extreme $\mathrm{pH}$, and elevated temperature. While all encapsulins showed the capacity to reassemble following $\mathrm{GuHCl}$-induced disassembly (within $75 \mathrm{~min}$ ), only the smallest $T=1$ nanocage reassembled after disassembly in basic $\mathrm{pH}$ (within $15 \mathrm{~min}$ ). Furthermore, atomic force microscopy revealed that all encapsulins showed a significant loss of structural integrity after undergoing sequential disassembly/reassembly steps. These findings provide insights into encapsulins' disassembly/reassembly dynamics, thus informing their future design, modification, and application.
\end{abstract}

KEYWORDS: encapsulin, protein nanocage, capsid assembly, self-assembly, intrinsic tryptophan fluorescence, atomic force microscopy 


\section{INTRODUCTION}

Protein nanocages (e.g., virus-like particles (VLPs), ferritins, heat-shock proteins) selfassemble from multiple protein subunits into highly-organised macromolecular structures, that exhibit well-defined inner cavities, outer surfaces, and interfaces between subunits. Their capacity to encapsulate cargo, coupled with the ability to genetically and/or chemically modify their structures, has enabled protein nanocages to be custom-engineered for a multitude of applications, including biocatalysis, materials synthesis, sensing, vaccines, and drug delivery. ${ }^{1}$

Encapsulins are an emerging class of protein nanocages found inside many archaea and bacteria. They self-assemble from identical protein subunits into hollow icosahedral nanocages that structurally resemble the major capsid protein gp5 of the HK97 virus. ${ }^{3,4}$ Based on their triangulation number $(T)$, all encapsulins exhibit one of the following three symmetrical icosahedral architectures: $T=1$ ( $24 \mathrm{~nm}, 60$-mer, 12 pentameric units); $T=3$ (32 nm, 180-mer, 12 pentameric and 20 hexameric units); and $T=4(42 \mathrm{~nm}, 240-\mathrm{mer}, 12$ pentameric and 30 hexameric units). ${ }^{5-8}$ In nature, encapsulins house cargo enzymes that mediate oxidative stress resistance, iron storage, anaerobic ammonium oxidation, or sulfur metabolism. ${ }^{9-11}$ Uniquely, encapsulins selectively self-assemble around cargo enzymes tagged with a small encapsulation signal peptide (ESig), packaging them. ${ }^{12}$ This mechanism has been adapted to load foreign cargo into encapsulins, reprogramming their functionality for different practical applications. $13-16$

Encapsulin subunits autonomously assemble, with extraordinary fidelity, into macromolecular nanocages. Such self-assembly is not only driven by folding of the individual polypeptide chains, but also by dynamic noncovalent interactions between the different polypeptide chains both within subunits and at the interfaces between subunits in the assembled supramolecular structure. ${ }^{17}$ Unravelling the self-assembly mechanisms of protein nanocages is complicated, especially if they exhibit highly symmetric homooligomeric structures, like encapsulins. $^{18}$ Nevertheless, multiple analytical techniques now allow the molecular mechanisms underlying protein nanocage assembly (e.g., protein folding) to be characterised and subsequently exploited.

For instance, the disassembly/reassembly of protein nanocages belonging to the ferritin family have been studied via a combination of: intrinsic tryptophan fluorescence (ITF), circular dichroism (CD), UV/VIS spectroscopy and synchrotron small-angle X-ray scattering (SAXS) measurements to assess protein conformation ${ }^{19-22}$, transmission electron microscopy (TEM) 
and dynamic light scattering (DLS) to evaluate structural integrity, shape and size distribution, and laser light scattering to monitor assembly kinetics. ${ }^{23}$ One study revealed that ferritin disassembles at extremely acidic $\mathrm{pH} 1.5$, then shows a rapid reassembly upon return to neutral $\mathrm{pH} 7.0$, accompanied by folding, followed by a slow phase in which the final 24-mer nanocage is formed. ${ }^{23}$ Importantly, this fundamental work led to the rational re-design of ferritin subunit interfaces, resulting in engineered nanocages capable of disassembly at a more amenable $\mathrm{pH}$ 4.0. ${ }^{24,25}$ Such modification now permits labile compounds (e.g. small-molecule drugs) to be controllably loaded into ferritin nanocages in a facile and non-destructive manner, enabling downstream applications (e.g., drug delivery). ${ }^{24,25}$

In contrast, experimental data pertaining to encapsulins' ability to disassemble/reassemble and the mechanisms that underpin this natural phenomenon are sparse. The most characterised system is the $T=1$ encapsulin from Thermotoga maritima (Tm-Enc), whose disassembly/reassembly has been primarily inspected via $C D$, polyacrylamide gel electrophoresis (PAGE), and TEM. ${ }^{14}$ Specifically, Tm-Enc has been found to disassociate in strong acidic and alkaline conditions, or high concentrations of denaturing agents (e.g., guanidine hydrochloride, GuHCl). Furthermore, Tm-Enc was shown to spontaneously reassemble upon returning to the initial conditions (i.e., neutral $\mathrm{pH}$ or absence of denaturant). ${ }^{27}$ Interestingly, $T m-E n c$ can be reassembled in the presence of ESig-tagged cargo (e.g., proteins, nanomaterials), resulting in their selective encapsulation in vitro, and thus further expanding encapsulins' utility. $8,27,2829$ Despite these promising findings, key questions concerning the biophysical mechanisms and physicochemical factors that underlie encapsulin disassembly/reassembly, and how they might be controlled, remain unanswered, especially for the $T=3$ and $T=4$ nanocages.

Motivated by this absence of information, we selected encapsulins with structures representing each of the three known architectures, and then interrogated their disassembly/reassembly. These nanocages included $T m-E n c(T=1)$, and the larger and more structurally complex encapsulins from Myxococcus xanthus $(M x-E n c, T=3)$ and Quasibacillus thermotolerans (Qt-Enc, $T=4)$. We combined intrinsic tryptophan fluorescence (ITF) spectroscopy, DLS, PAGE and TEM, to accurately monitor the assembly states of all three encapsulins under varying physicochemical conditions, including exposure to extreme $\mathrm{pH}$, strong denaturants $(\mathrm{GuHCl})$, and elevated temperatures. Furthermore, the effect disassembly/reassembly had on the nanocages' structural integrity was evaluated by atomic force microscopy (AFM). Together, this work provides critical insights into the dynamic 
mechanisms that govern the disassembly/reassembly of differing encapsulin structures, which will help to expedite and broaden their future design, modification, and practical application.

\section{MATERIALS AND METHODS}

\section{Materials}

All chemicals and reagents used in this study were purchased from Sigma-Aldrich, unless stated otherwise.

\section{Molecular cloning of constructs}

All inserts were codon optimised for expression in Escherichia coli and custom synthesised as gBlock Gene Fragments (Integrated DNA Technologies). Encapsulins from Thermotoga maritima (Tm) (UniProt: TM_0785), Myxococcus xanthus (Mx) (UniProt: MXAN_3556) and Quasibacillus thermotolerans (Qt) (UniProt: QY95_01592) were each synthesised with flanking restriction sites (NcoI/BamHI). For gene expression in E. coli, Tm-Enc was cloned into pETDuet-1 (Novagen, Merck), and $M x-E n c$ and Qt-Enc were cloned into pACYC-Duet-1 (Novagen, Merck), summarised in Supplementary Table S1. E. coli $\alpha$-Select (Bioline, UK) was used for general plasmid storage and propagation. Gene insertion was confirmed by PCR using primer pairs pETUpstream/DuetDOWN (Merk). E. coli BL21 (DE3) cells (New England Biolabs) were used for recombinant protein expression. Herein, cells were transformed with the appropriate plasmids, and the resulting transformants were selected on Luria-Bertani (LB) agar supplemented with either $100 \mathrm{mg} / \mathrm{mL}$ of carbenicillin or $50 \mathrm{mg} / \mathrm{mL}$ of chloramphenicol (see Supplementary Table S1).

\section{Recombinant Protein Production}

Protein expression experiments were performed in LB medium supplemented with carbenicillin $(100 \mathrm{mg} / \mathrm{mL})$ or chloramphenicol $(50 \mathrm{mg} / \mathrm{mL})$. Briefly, strains were streaked out on LB agar plates and grown overnight at $37^{\circ} \mathrm{C}$. A starter culture ( 1 colony in $5 \mathrm{~mL} \mathrm{LB}$ ) was grown for $16 \mathrm{~h}$ at $37^{\circ} \mathrm{C}$ and used to inoculate $500 \mathrm{~mL}$ of LB media. Cultures were incubated at $37^{\circ} \mathrm{C} / 200-250 \mathrm{rpm}$ until an optical density at $600 \mathrm{~nm}$ (OD600) of 0.5-0.6 was reached. Protein synthesis was then induced by the addition of isopropyl- $\beta$-d-thiogalactopyranoside (IPTG) to a final concentration of $0.1 \mathrm{mM}$. Induced cultures were incubated at $37^{\circ} \mathrm{C} / 200-250 \mathrm{rpm}$ for 4 
$\mathrm{h}$ and then cells were harvested via centrifugation $\left(8,000 \times \mathrm{g}, 4^{\circ} \mathrm{C}, 15 \mathrm{~min}\right)$. The resulting cell pellets were stored at $-30^{\circ} \mathrm{C}$ until further use.

\section{Protein purification}

Cell pellets from $500 \mathrm{~mL}$ encapsulin-producing cultures were thawed and resuspended in 30 $\mathrm{mL}$ of lysis buffer (50 mM 4-(2-hydroxyethyl)-1-piperazineethanesulfonic acid (HEPES) buffer pH 7.4 (Chem-Supply Pty) and Benzonase ${ }^{\circledR}$ nuclease $10 \mathrm{U} / \mathrm{mL}$ ). Cells were lysed by three rounds of passage through a French pressure cell at $1000 \mathrm{psi}$ and centrifuged at 8,000 $\mathrm{x}$ $g, 4^{\circ} \mathrm{C}$ for $15 \mathrm{~min}$. Supernatant containing soluble protein was heat treated in a water bath at $65^{\circ} \mathrm{C}$ for $15 \mathrm{~min}$ before centrifugation $\left(10,000 \times \mathrm{g}, 4^{\circ} \mathrm{C}, 10 \mathrm{~min}\right)$. Protein precipitation was initiated by adding $10 \%(\mathrm{w} / \mathrm{v})$ PEG8000 and $2 \%(\mathrm{w} / \mathrm{v}) \mathrm{NaCl}$ to the supernatant, followed by incubation on ice for $30 \mathrm{~min}$. Next, the sample was spun down at $10,000 \mathrm{xg}$ for $10 \mathrm{~min}$ at $4{ }^{\circ} \mathrm{C}$. The precipitated protein was resuspended in $2.5 \mathrm{~mL}$ of HEPES buffer $(50 \mathrm{mM}, \mathrm{pH} 7.4)$ and filtered through a $0.22 \mathrm{~mm}$ syringe filter.

All purifications were carried out on an Äkta ${ }^{\mathrm{TM}}$ start chromatography system (GE Healthcare). The three encapsulins used in this study were purified via size exclusion chromatography (SEC) using a HiPrep 26/60 Sephacryl S-500 HR column (GE Healthcare) equilibrated with $50 \mathrm{mM}$ HEPES pH 7.4. Fractions showing the corresponding encapsulin band on SDS-PAGE were pooled and subjected to further purification via anion-exchange chromatography using a HiPrep Q 16/10 column (GE Healthcare) equilibrated with $50 \mathrm{mM}$ HEPES pH 7.4. Encapsulin proteins were eluted with linear gradient of $0-0.3 \mathrm{M} \mathrm{NaCl}$ and $0.3-$ $1 \mathrm{M} \mathrm{NaCl}$ (Supplementary Figure S1). Fractions containing encapsulin, identified via sodium dodecyl sulphate polyacrylamide gel electrophoresis (SDS-PAGE), were pooled, concentrated and buffer exchanged into $50 \mathrm{mM}$ HEPES buffer $\mathrm{pH} 7.4$ using Amicon Ultra-15 centrifugal filter units with a $100 \mathrm{KDa}$ cut-off. Lastly, purified encapsulin Enc proteins were filtered through a $0.22 \mathrm{~mm}$ syringe filter and stored at $-30^{\circ} \mathrm{C}$ until further use.

\section{PAGE analysis and protein quantification}

Protein samples were denatured, separated, and visualised using SDS-PAGE, with molecular weights compared with a commercial protein ladder (Precision Plus Protein, BioRad). The BioRad mini-protean system (Bio-Rad laboratories) was used for SDS-PAGE analysis. Protein samples were mixed in a 1:1 ratio with $2 \mathrm{X}$ Laemmli sample buffer with $50 \mathrm{mM}$ 1,4dithiothreitol and heated at $99^{\circ} \mathrm{C}$ for $10 \mathrm{~min}$ at $300 \mathrm{rpm}$. Electrophoresis was performed at 200 
V for 30 min on a 4-20\% polyacrylamide gel (Mini-PROTEAN® TGX ${ }^{\mathrm{TM}}$, BioRad) in SDS running buffer (25 mM Tris, $192 \mathrm{mM}$ glycine, 1\% (w/v) SDS, pH 8.3). Gels were stained following the Coomassie G-250 safe stain protocol. Encapsulin assembly was visualised via non-denaturing Blue Native-PAGE (BN-PAGE). The XCell SureLock Mini-Cell Electrophoresis System (ThermoFisher Scientific) was used for BN-PAGE analysis. Protein samples were mixed in a 1:4 ratio with $4 \mathrm{X}$ Native-PAGE sample buffer (ThermoFisher Scientific) and loaded into NativePAGETM 3-12\% Bis-Tris protein gels (ThermoFisher Scientific). BN-PAGE was performed using two different running buffers: $1 \mathrm{X}$ anode buffer (NativePAGETM running buffer 20X, ThermoFisher Scientific) in the outer buffer chamber and $1 \mathrm{X}$ dark blue cathode buffer (1X anode buffer, $0.02 \%(\mathrm{w} / \mathrm{v})$ Coomassie G-250) in the inner buffer chamber. Lastly, the samples were run on ice at $150 \mathrm{~V}$ for $90 \mathrm{~min}$ follow by a second run at $250 \mathrm{~V}$ for $30 \mathrm{~min}$. Protein concentration was determined by measuring the absorbance at $280 \mathrm{~nm}$ on a NanoDrop 2000 Spectrophotometer instrument (ThermoFisher Scientific).

\section{In vitro disassembly/reassembly of encapsulin}

To characterise the in vitro disassembly of encapsulin, the presence of a denaturing agent $(\mathrm{GuHCl})$, changes in $\mathrm{pH}$, and thermally induced disassembly methods were used. GuHCl was added to the encapsulin sample to final concentrations between 1-7 M. For $\mathrm{pH}$ disassembly, dilution of the purified encapsulins in $50 \mathrm{mM}$ HEPES at different $\mathrm{pH}$ (3-13) was performed. Final $\mathrm{pH}$ was confirmed with $\mathrm{pH}$ strips. Once in their respective conditions, encapsulin samples were incubated for $1 \mathrm{~h}$ at room temperature. For thermally induced disassembly, the encapsulin sample was subjected to a temperature ramp from $20-90^{\circ} \mathrm{C}$ at a rate of $2^{\circ} \mathrm{C} / \mathrm{min}$. In all disassembly methods, the final encapsulin concentration was $5 \mu \mathrm{M}$, dithiothreitol (DTT) was added to the encapsulin sample to a final concentration of $1 \mathrm{mM}$ and $50 \mathrm{mM}$ HEPES pH 7.4 was used to reach the desired final volume.

For stability experiments, $5 \mu \mathrm{M}$ of encapsulin was incubated for $1 \mathrm{~h}$ at room temperature under reducing (10 $\mathrm{mM} \mathrm{DTT})$, oxidising $\left(10 \mathrm{mM} \mathrm{H}_{2} \mathrm{O}_{2}\right)$, and high ionic strength $(1 \mathrm{M} \mathrm{NaCl})$ conditions.

The subsequent reassembly of encapsulins was initiated by returning the sample to original conditions. Briefly, the samples were dialysed against 50 mM HEPES buffer $\mathrm{pH} 7.4$ and $1 \mathrm{mM}$ DTT at room temperature overnight, using 3.5K MWCO SnakeSkin dialysis tubing (ThermoFisher Scientific). To measure the reassembly rate, samples were removed from 
dialysis every $15 \mathrm{~min}$ for $75 \mathrm{~min}$ and centrifuged for $5 \mathrm{~min}$ at $10,000 \mathrm{x} g$ to remove any aggregated proteins.

\section{Intrinsic tryptophan fluorescence}

ITF measurements of encapsulins in their varying states of assembly were performed with an FP-8500 Spectrofluorometer (JASCO) using a $3 \mathrm{~mm}$ pathlength micro-volume quartz cuvette. Samples $(60 \mu \mathrm{L})$ were prepared in triplicate with a final encapsulin concentration of 5 $\mu \mathrm{M}$. Samples were excited at $290 \mathrm{~nm}$ and emission spectra were collected from 300-450 nm. The measurement parameters were: excitation and emission bandwidths of $5 \mathrm{~nm}$, response of $0.2 \mathrm{~s}$, medium sensitivity, data interval of $0.1 \mathrm{~nm}$, scan speed of $100 \mathrm{~nm} / \mathrm{min}$, and 4 measurement accumulations were averaged. To investigate the effect of temperature on the different encapsulins, spectra were collected from $20-90^{\circ} \mathrm{C}$ with a temperature ramp of $2^{\circ} \mathrm{C} / \mathrm{min}$. The obtained spectra were further processed by buffer spectra subtraction using Spectra Manager ${ }^{\mathrm{TM}}$ software (JASCO), and the ratio between the fluorescence intensity at 360 and $330 \mathrm{~nm}$ (360/330) was calculated and plotted in Microsoft Excel.

\section{Transmission electron microscopy (TEM)}

To visualise morphology, size, and state of encapsulin assembly, TEM was performed using a Philips CM10 microscope (100 kV accelerating voltage). Briefly, encapsulin samples (0.2 $\mathrm{mg} / \mathrm{mL}$ ) were adsorbed onto pioloform-coated 200 mesh copper grids (ProSciTech) for $2 \mathrm{~min}$. Prior to imaging, samples were negatively stained for $1 \mathrm{~h}$ using uranyl acetate replacement (UAR-EMS, Electron Microscopy Sciences), washed with ultrapure water, and allowed to dry for at least $15 \mathrm{~min}$.

\section{Dynamic light scattering}

To measure the diameter of encapsulins, DLS was performed with a Malvern Zetasizer ZSP instrument equipped with a $633 \mathrm{~nm}$ laser. Samples with a final encapsulin concentration of $5 \mu \mathrm{M}$ were prepared as described above. Three measurements were performed at $25^{\circ} \mathrm{C}$, using a plastic microcuvette (ZEN0040, Malvern), $173^{\circ}$ backscatter and automatic attenuator selection. Data analysis was performed in the Zetasizer Nano software. All DLS sizes reported, herein, are size by number values calculated using distribution analysis. A $1 \mathrm{~cm}$ quartz cuvette was used for temperature ramp experiments. Heat maps were made using GraphPad Prism 9. 


\section{Atomic Force Microscopy (AFM)}

Images and force curve measurements were obtained using a Cypher ES Atomic Force Microscope (Oxford Instrument, Asylum Research, Santa Barbara, CA, USA) at room temperature $\left(25^{\circ} \mathrm{C}\right)$. Protocols were adapted from Collett et $a l^{26,27}$. For imaging, the instrument was operated in amplitude modulated-AFM (AM-AFM), while force measurements were obtained in contact mode. Biolever BL-AC40TS cantilevers (Oxford Instruments, Asylum Research, Santa Barbara, CA, USA, nominal spring constant $\mathrm{k}_{\mathrm{c}}=0.09 \mathrm{~N} / \mathrm{m}$ ) were used for all measurements. All experiments were completed within a droplet of media $(\sim 100 \mu \mathrm{L})$ with a concentration of $\sim 100 \mathrm{ng}$ of encapsulin deposited onto freshly cleaved muscovite surface (the supporting substrate). Prior to experimentation, each cantilever was calibrated via the thermal spectrum method and the lever sensitivity was determined using force spectroscopy. Processing of AFM data involved using a combination of the Asylum Research software, custom MATLAB codes, and the Gwyddion software package ${ }^{28}$.

\section{Determining elasticity of intact encapsulins}

Force versus distance curves (FD curves) were obtained from the central region of encapsulins. Following image location, FD curves were first obtained on an area of bare mica next to the nanocage to ensure linear (non-elastic) FD curves were observed, which provided a reference for determining elasticity of the particles. The spring constant $\left(\mathrm{k}_{\mathrm{c}}\right)$ of the cantilever was determined for each cantilever used as described above (values in the range of 0.05-0.1 $\mathrm{N} / \mathrm{m}$ were obtained for all cantilevers). The tip was then directed to the central region of the particle to obtain accurate indentation data. Upward of 50 FD curves were recorded across several particles for each sample.

The tip-sample contact point between the AFM cantilever and the encapsulin was performed independently for each FD curve analysed using methods previously described ${ }^{29}$. Specifically, the contact point between the AFM cantilever tip and the encapsulin is defined as the point at which the cantilever first makes physical contact with the surface adsorbed particle. Following this point, the cantilever bends in response to interactions with the encapsulin, while the nanocage itself is indented. In a raw FD curve (z-displacement $(\mathrm{Z})$ vs. cantilever deflection (d)), this point of first contact is mathematically defined as $Z_{0}$ and $d_{0}$, respectively. This is observed in the AFM force curve as an increase in force (y-axis), above the zeroed baseline, as a function of distance ( $\mathrm{x}$-axis prior to zeroing the indentation).

The indentation $(\delta)$ is then calculated via: 


$$
\delta=\left(\mathrm{z}-\mathrm{z}_{0}\right)-\left(\mathrm{d}-\mathrm{d}_{0}\right)(1)
$$

and transformed into indentation vs. force curves using Hooke's law;

$$
\mathrm{F}=\mathrm{K}_{\mathrm{c}} \mathrm{xd}(2)
$$

The elastic response of the samples is then fit to the Hertz/Sneddon equation (3) to obtain a Young's elastic modulus $(E)$ for each of the fitted curves. Using custom MATLAB scripts, curves were fitted to the equations:

$$
F_{\text {cone }}=\frac{2}{\pi} \tan \alpha E^{*} \delta^{2}(3)
$$

and;

$$
E^{*}=\frac{E}{1-v^{2}}(4)
$$

where $\mathrm{F}$ is loading force, $\delta$ is indentation depth, $\alpha$ is the cone opening angle, $\mathrm{E}^{*}$ is the apparent elastic modulus, and $\mathrm{v}$ is the Poisson's ratio. A conal tip angle of $34.4^{\circ}$ and a Poisson's ratio of 0.5 was used for processing of all force curves. All elasticity values were obtained from curves fitted with an $\mathrm{R}^{2}$ value of 0.9 or above. Data falling below this quality was rejected for further analysis. 


\section{RESULTS AND DISCUSSION}

\section{Monitoring encapsulin assembly/disassembly using intrinsic tryptophan fluorescence}

Unloaded Tm-Enc, $M x-E n c$, and $Q t-E n c$ were produced in E. coli and purified by SEC and anion exchange chromatography prior to biophysical characterisation. Purification and correct self-assembly were confirmed using SDS-PAGE, blue native-PAGE, DLS, and TEM (Figure $2 \mathrm{~g}$, Supplementary Figure S1). TEM images of self-assembled Tm-Enc, $M x$-Enc, and $Q t-E n c$ displayed structures with consistent shape and size (Figure 2g) and DLS analysis indicated a diameter of $23.7 \pm 4.9 \mathrm{~nm}$ for $T m-E n c$, consistent with its crystal structure data ${ }^{12}$, and a diameter of $32.0 \pm 6.0 \mathrm{~nm}$ for $M x-E n c$ and $38.2 \pm 7.3 \mathrm{~nm}$ for $Q t$-Enc, consistent with their Cryo-EM structures. ${ }^{6,8} M x$-Enc was produced primarily in its $T=3$ structure; however, populations of smaller, $T=1$ like, structures were evident in Native-PAGE and TEM results $(\sim 18 \mathrm{~nm})$ (Supplementary Figure S1, Figure $2 \mathrm{~g}$ ). This variation in size of $M x$-Enc has been previously observed, where recombinantly produced $M x$-Enc without the presence of ESigtagged cargo will form heterogenous $T=1$ and $T=3$ populations. ${ }^{8}$

Tryptophan fluorescence is extremely sensitive to local environment. As such, protein unfolding, disassembly or conformational transitions often result in a change in emission spectra of the $\operatorname{Trp}(\mathrm{s})$ within a protein; a lower maximum wavelength (blue-shifted) when the $\operatorname{Trp}(\mathrm{s})$ are buried, and a higher maximum wavelength (red-shifted) when solvent exposed. ${ }^{30}$

Encapsulin subunits adopt a HK97-fold and have three conserved structural regions, a peripheral (P)-domain, axial (A)-domain, and an elongated (E)-loop region (Supplementary Figure S2). ${ }^{3,4}$ Conveniently, each subunit of $T m-E n c, M x$-Enc, and $Q t-E n c$ contains five, three, and two Trp residues, respectively. For each encapsulin, at least one of these Trps is located within the interface between subunits, and one is located within the hydrophobic core of a single subunit (Figure 1a, b \& c). Therefore, these intrinsic Trp residues are likely to be suitable reporters for both assembly/disassembly of the encapsulin macrostructure and folding of the individual subunit polypeptide chains. Indeed, intrinsic Trp fluorescence (ITF) has previously been used to monitor the re-folding of $T=1$ encapsulin from Rhodococcus erythropolis $\mathrm{N771}$ when desorbed from a zeolite substrate. ${ }^{31}$ ITF is also an appealing technique to monitor the process of encapsulin assembly/disassembly owing to its relative simplicity, ability to report on a dynamic ensemble of structures in solution and non-destructive nature, which enables measurements to be performed in real-time. Additionally, unlike fluorescence resonance energy transfer (FRET)-based techniques, ITF does not require modification of the protein with any extrinsic labels that may alter the assembly/disassembly dynamics. Although circular 
dichroism (CD) may provide more specific secondary structural information, unlike ITF, CD is not compatible with the high concentration of $\mathrm{GuHCl}$ and extreme $\mathrm{pH}$ likely required for encapsulin disassembly. ${ }^{32}$

Figure 1 shows the ITF of assembled Tm-Enc, $M x$-Enc and $Q t$-Enc overlayed with the spectra obtained after $1 \mathrm{~h}$ incubation with $7 \mathrm{M} \mathrm{GuHCl}$. For all three assembled encapsulins, blueshifted Trp fluorescence spectra are observed ( $\sim 334 \mathrm{~nm}$ maximum emission wavelength for Tm-Enc and Qt-Enc; and $\sim 338 \mathrm{~nm}$ for $M x-E n c)$, while in the presence of $7 \mathrm{M} \mathrm{GuHCl}$, a dramatic red-shift of the fluorescence spectra is seen $(\sim 354 \mathrm{~nm}$ maximum emission wavelength). A maximum emission wavelength over $350 \mathrm{~nm}$ is observed where the Trps within a protein are completely solvent exposed, as in an unfolded polypeptide chain. ${ }^{33}$ Therefore, it appears that $7 \mathrm{M} \mathrm{GuHCl}$ fully disassembles $T m$-Enc, as has previously been demonstrated ${ }^{14}$, as well as both $M x$-Enc and $Q t-E n c$, by completely unfolding the protein.

It is also interesting to note that the overall fluorescence emission spectral shape represents an average of all Trp environments present in the protein. ${ }^{34}$ Where a protein contains multiple Trps, these may exist in different environments and therefore emit at different wavelengths. While we cannot draw conclusions about the foldedness/assembly of Tm-Enc, Mx-Enc and QtEnc relative to one another (as they all contain a different number of Trps), changes in the spectral shape for the same encapsulin may discern encapsulin assembly (i.e., interaction between subunits) as opposed to the folding of individual subunit polypeptide chains. This is particularly true for Qt-Enc. As can be seen in Figure 1f, the peak-shape for assembled Qt-Enc is flattened. This is consistent with its two Trp residues being located in different solvation environments such that the spectra two Trps overlap, but are not resolved (i.e., Trp95 being more buried in a helix at the pentamer/hexamer interface than Trp154, which is located on a loop in the A-domain, nestled within the hydrophobic core of a single Qt-Enc subunit) (Figure 1c, Supplementary Figure S2).

Instead of reporting the maximum fluorescence wavelength, herein, we have presented much of our ITF data as a fluorescence intensity ratio of emission at $360 \mathrm{~nm}$ and $320 \mathrm{~nm}$ (360/320). This ratio is used as a proxy for the shift in overall Trp fluorescence emission peak, and avoids any bias arising from the spectral peak shape. ${ }^{35}$ This approach has allowed us to expand the repertoire of methods used to monitor encapsulin assembly/disassembly across a range of conditions. 
a.

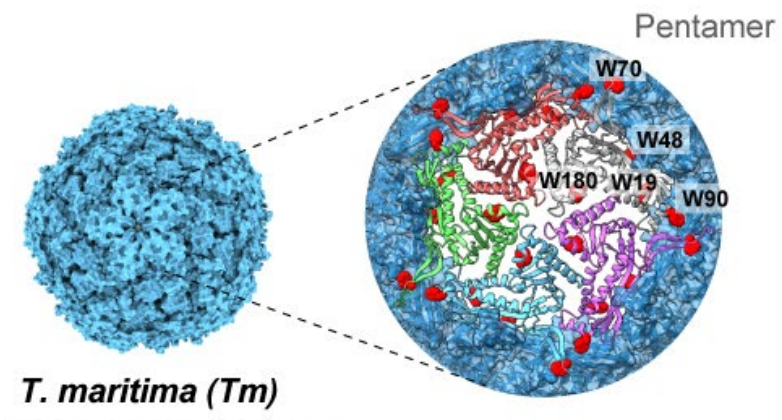

$T=1 ; 60$ subunits; $20-24 \mathrm{~nm}$

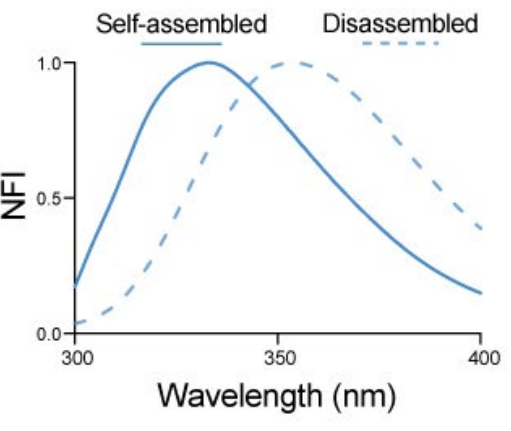

b.
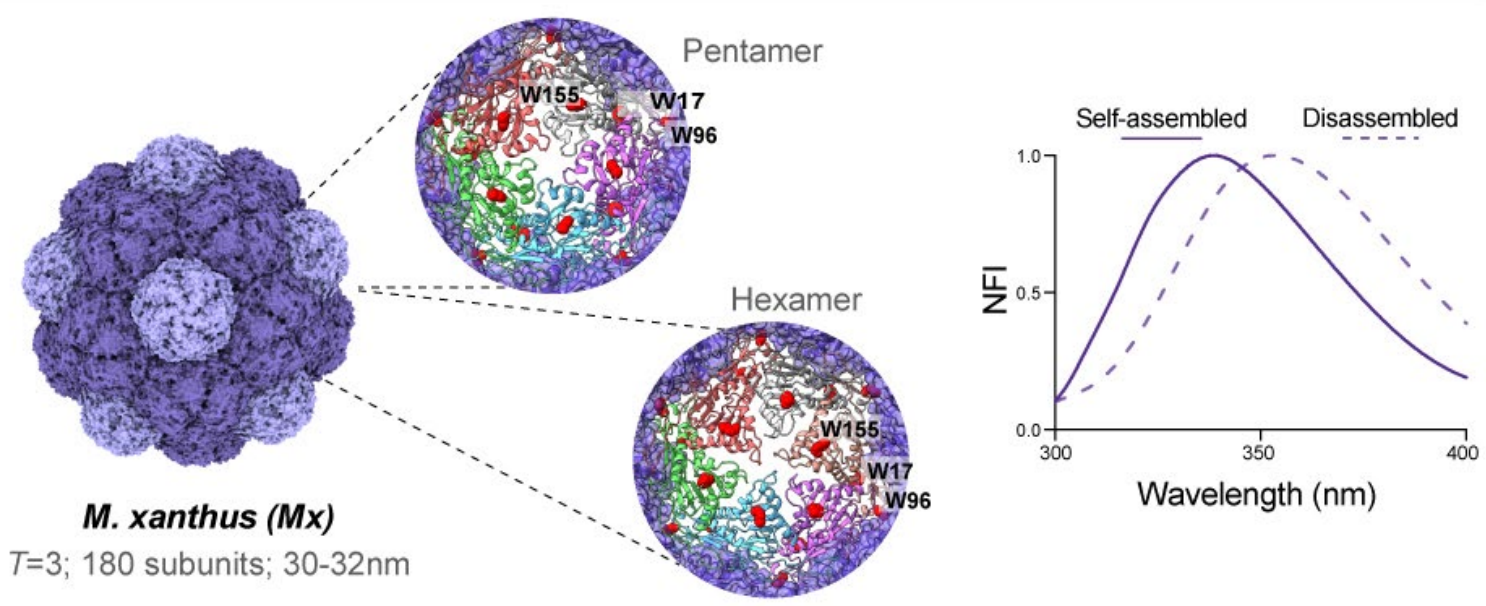

C.
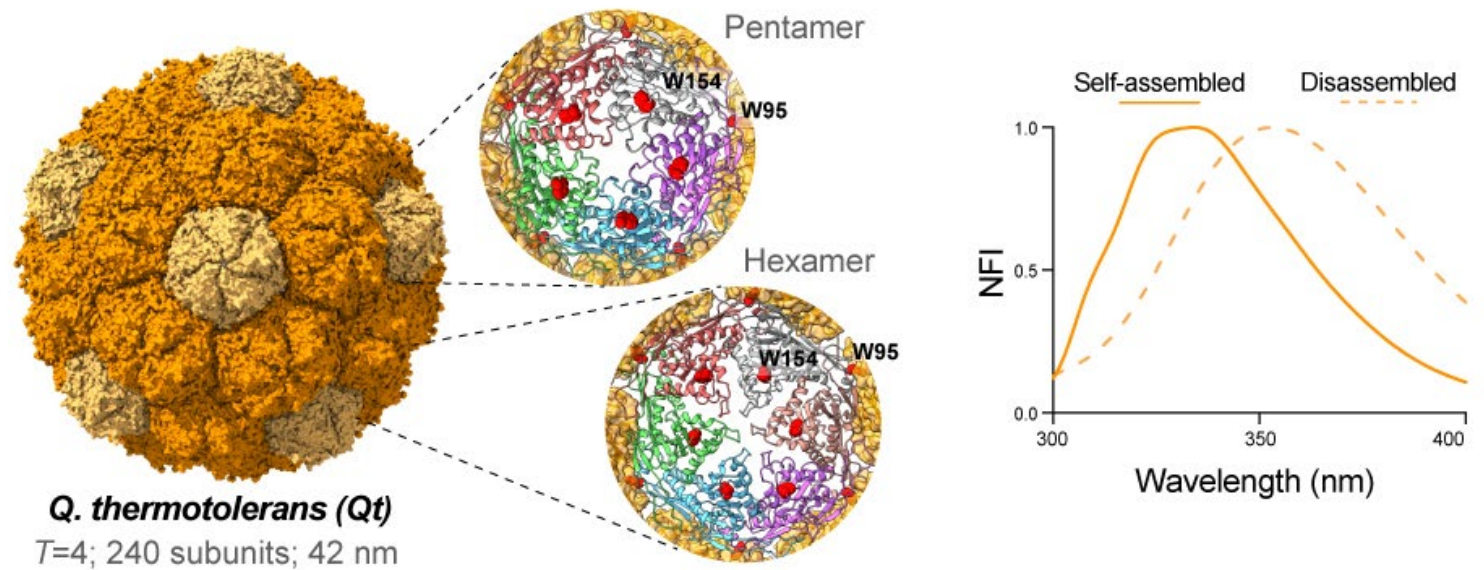

Figure 1. Monitoring encapsulin nanocage assembly states via ITF. Schematic diagram showing the assembled architectures of (a) Tm-Enc (PDB: 3DKT), (b) $M x$-Enc (PDB: 4PT2), and (c) Qt-Enc (PDB: 6NJ8). For each structure, the pentameric and hexameric units are shown in light and saturated colours, respectively. Within the expanded pentameric or hexameric units, the tryptophan (W) residues belonging to individual subunits are highlighted in red: Tm: W19, W48, W70, W90 and W180; $M x$ : W17, W96 and W155; Qt: W95 and W154. Molecular graphics were created using UCSF ChimeraX ${ }^{36}$. Using ITF, a shift in emission discriminated between the maximum emission wavelength of buried Trp in 'assembled' form: $\sim 334 \mathrm{~nm}$ for (a) $T m-E n c$ and (c) $Q t-E n c$, or $\sim 338$ for (b) $M x$-Enc; and solvent exposed Trp in 'disassembled' form $(\sim 354 \mathrm{~nm})$ upon addition of $7 \mathrm{M} \mathrm{GuHCl}$. Emission wavelengths are represented as Normalised Fluorescence Intensity (NFI). 


\section{GuHCl induced encapsulin disassembly}

$\mathrm{GuHCl}$ is a denaturant that affects protein structure by disrupting non-covalent interactions, including hydrogen bonding and hydrophobic effects. ${ }^{37}$ Our initial ITF results indicated that $7 \mathrm{M} \mathrm{GuHCl}$ completely unfolds the Tm-Enc, $Q t-E n c$ and $M x$-Enc protein polypeptide chains (Figure 1), and thus, disassembles the encapsulin macrostructure. To further examine the effect of $\mathrm{GuHCl}$ on encapsulin folding and assembly, all encapsulins were incubated in varying concentrations of $\mathrm{GuHCl}(0-7 \mathrm{M})$ for $1 \mathrm{~h}$ before performing ITF and DLS.

The observed shift in Trp emission peak for Tm-Enc, $M x-E n c$ and $Q t-E n c$ in the presence of $\mathrm{GuHCl}$ are shown in Figure 2a. As expected from our previous results, all three encapsulins show a blue to red-shift from the assembled $(0 \mathrm{M} \mathrm{GuHCl}, \Delta 360 / 320=0)$ to unfolded state $(7 \mathrm{M}$ $\mathrm{GuHCl}, \Delta 360 / 320=1)$. However, the concentration of $\mathrm{GuHCl}$ at which this transition occurs varies significantly between the different encapsulins. Tm-Enc Trp solvation began from approximately $3 \mathrm{M} \mathrm{GuHCl}$, with complete Trp solvation from $6 \mathrm{M} \mathrm{GuHCl,} \mathrm{while} M x$-Enc and Qt-Enc Trp solvation began at a lower concentration of $1 \mathrm{M} \mathrm{GuHCl}$, with complete Trp solvation from $4 \mathrm{M}$ and $5 \mathrm{M} \mathrm{GuHCl}$, respectively. This variation may reflect structural complexity, with the smaller and pentameric Tm-Enc being the most robust to $\mathrm{GuHCl}$ compared to the larger pentameric and hexameric $M x$-Enc and Qt-Enc.

To complement these ITF results, DLS analysis was performed to characterise the size distribution of encapsulin in solution for the same samples (Figure 2b, Supplementary Table S2). For $M x-E n c$, no intact encapsulin macrostructure is detectable by DLS at concentrations $\geq 3 \mathrm{M} \mathrm{GuHCl}$, which also correlates with the significant red-shifted Trp emission at $3 \mathrm{M}$ $\operatorname{GuHCl}(\Delta 360 / 320=0.88)$. This result suggests that $M x-E n c$ is both disassembled and largely unfolded at $\mathrm{GuHCl}$ concentrations $\geq 3 \mathrm{M}$. However, for Tm-Enc and Qt-Enc, absence of the intact encapsulin macrostructure occurs at lower $\mathrm{GuHCl}$ concentrations than the major redshift in Trp emission. For example, at $4 \mathrm{M} \mathrm{GuHCl}$, the Tm-Enc macrostructure is not detectable by DLS, but the Trp emission remains significantly blue-shifted $(\Delta 360 / 320=0.20)$, corresponding to a native-like fold. A similar result is seen for $Q t-E n c$ at $3 \mathrm{M} \mathrm{GuHCl}(\Delta 360 / 320$ $=0.27)$. TEM and native-PAGE images of each encapsulin after incubation in the lowest $\mathrm{GuHCl}$ concentration required for significant Trp solvation also show the absence of nanocage macrostructures, confirming their disassembly. Upon dialysis in buffer (50 mM HEPES, $1 \mathrm{mM}$ DTT) overnight, all encapsulins subsequently reassembled into their original macrostructure (Figure 2h, Supplementary Figure S3). 
Taken together, these results point to the existence of an intermediate state(s) in $T m$-Enc and Qt-Enc GuHCl-induced disassembly, in which the nanocage breaks down into smaller $(<0.5$ $\mathrm{nm}$ ) entities with a native-like fold. There is insufficient evidence from ITF or DLS to define the stoichiometry or structure of this intermediate state(s). However, a previous native mass spectrometry study of the $T=1$ encapsulin from $B$. linens suggested reassembly occurs via formation of stable dimers prior to the final nanocage. ${ }^{38}$ It is possible that disassembly of $\mathrm{Tm}$ Enc and Qt-Enc proceeds via a similar mechanism. We also cannot rule out that $M x-E n c$ proceeds via an equivalent intermediate state upon GuHCl-induced disassembly. However, if this is the case, our data suggests the chemical stability of the nanocage and the intermediate for $M x-E n c$ are more closely matched, and less chemically stable, than those of Tm-Enc or $Q t$ Enc, such that both disassembly and complete unfolding occur at close to $3 \mathrm{M} \mathrm{GuHCl}$. This potential model of encapsulin disassembly warrants further investigation, as disassembly of the nanocage utilising lower $\mathrm{GuHCl}$ concentrations, without completely unfolding the encapsulin protein, may provide an effective scheme for loading and/or releasing more chemically sensitive cargo.

Overall, our results for GuHCl-induced encapsulin disassembly suggest that folding plays a critical role for encapsulin nanocage assembly/disassembly. The smaller, less complex Tm-Enc structure requires more $\mathrm{GuHCl}$ for unfolding than the larger, more complex $Q t$-Enc and $M x$ Enc. This agrees with the difference in the high stability of ferritin, which is a small protein nanocage comprised of 24 subunits, compared to the high sensitivity of larger and more structurally complex VLPs in denaturants. Ferritin is stable up to $6 \mathrm{M} \mathrm{GuHCl}^{39}$, and requires 8 $\mathrm{M}$ of denaturant urea for disassembly. ${ }^{40} \mathrm{~A}$ concentration of $2 \mathrm{M} \mathrm{GuHCl}$ is not strong enough to disassemble ferritin, but is able to unfold the $\alpha$-helices surrounding ferritin channels, thereby enlarging its pores to allow entry of cargo without the need for complete disassembly. ${ }^{41}$ Contrastingly, the larger P22 VLP, which assembles from 420 subunits into a $T=7$ nanoparticle-like structure that is $56 \mathrm{~nm}$ in diameter, dissociates at just $3 \mathrm{M} \mathrm{GuHCl} .{ }^{42}$ However, the chemical stability of the encapsulins tested is not directly related to their size, as $Q t$-Enc requires more $\mathrm{GuHCl}$ for unfolding than the smaller $M x$-Enc. Hence, other factors, such as the symmetry and stability of intermediate state(s), also needs to be taken into consideration.

\section{pH induced encapsulin disassembly}

To investigate the role of electrostatic interactions in maintaining encapsulin macrostructure, ITF and DLS were also performed for the three encapsulins at varying $\mathrm{pH}$ (Figure $2 \mathrm{c} \& \mathrm{~d}$ ). The 
theoretical $\mathrm{pI}$ of $T m-E n c, M x-E n c$ and $Q t-E n c$ are $4.90,5.45$ and 5.02, respectively. ${ }^{43}$ If electrostatic interactions play a major role in the mechanism of encapsulin assembly, we would expect to see disassembly of the encapsulin nanocage at $\mathrm{pH}<\mathrm{pI}$ (where the overall charge on the protein would be positive rather negative, as at neutral $\mathrm{pH}$ ). However, this is not the case. ITF indicates that all encapsulins remain relatively stable across a broad $\mathrm{pH}$ range, with a significant change in Trp exposure only observed in extreme alkaline conditions ( $\mathrm{pH}$ 12-13) (Figure 2c). DLS demonstrates the presence of assembled encapsulin at all pHs, with the exception of $\mathrm{pH} 13$, and smaller structures $(\sim 13 \mathrm{~nm})$ at $\mathrm{pH} 12$ for $M x$-Enc (Figure 2d, Supplementary Table S3). Disassembly of the nanocage structures at $\mathrm{pH} 13$ was also confirmed by TEM (Figure 2i). Therefore, loss of electrostatic interactions between and/or within encapsulin subunits is unlikely to be a major driving force for disassembly. Disassembly more likely arises at extreme alkaline $\mathrm{pH}$ due to deprotonation of the protein side chains, resulting in a loss of the hydrogen bonding that holds the protein scaffold together. ${ }^{44}$

\section{Thermal encapsulin disassembly}

To assess encapsulin thermostability, assembled samples were heated to $90^{\circ} \mathrm{C}$ with ITF and DLS measurements taken every $10^{\circ} \mathrm{C}$ up to $90^{\circ} \mathrm{C}$, as well as upon return to $20^{\circ} \mathrm{C}$. Additionally, encapsulins were heated to $90^{\circ} \mathrm{C}$ with TEM samples prepared both immediately after heating, and also after being cooled at $20^{\circ} \mathrm{C}$ for $1 \mathrm{~h}$. ITF results showed Tm-Enc encapsulin remained stable throughout heating, with only a very slight observed red-shift in emission wavelength at temperatures above $80^{\circ} \mathrm{C}$, which was recovered when cooled back to $20^{\circ} \mathrm{C}$ (Figure 2e). No significant change in size of the Tm-Enc nanocages was observed by DLS, and TEM images showed assembled cages after heating to $90^{\circ} \mathrm{C}$, suggesting that the $T m$-Enc nanocage is resistant to thermal disassembly (Figure $2 \mathrm{f} \& \mathrm{j}$, Supplementary Table S4).

In contrast to $T m-E n c$, both $M x-E n c$ and $Q t-E n c$ disassemble at elevated temperatures. $M x$ Enc Trp solvation began from $60^{\circ} \mathrm{C}$ and disassembled from $80^{\circ} \mathrm{C}$, as shown by DLS (Figure 2e \& f, Supplementary Table S4), and appeared disassembled at $90^{\circ} \mathrm{C}$ via TEM imaging (Figure $2 \mathrm{j}$ ). Additionally, in contrast to Tm-Enc, disassembly of $M x$-Enc by temperature is not reversible, as when cooled back to $20^{\circ} \mathrm{C}$ only large aggregates were found (Figure $2 \mathrm{f}$, Supplementary Table S4). Similarly, ITF showed Qt-Enc began Trp solvation from $40^{\circ} \mathrm{C}$ and DLS indicated the majority of sample disassembled at $80^{\circ} \mathrm{C}$ and $90^{\circ} \mathrm{C}$. However, even at $90^{\circ} \mathrm{C}$, a smaller population (approx. 16\%) of Qt-Enc subunits remained in intermediate structures of $\sim 13 \mathrm{~nm}$ (Figure 2f, Supplementary Table S4). When cooled back to $20^{\circ} \mathrm{C}$, DLS and TEM 
indicated Qt-Enc reassembled to its original size and ITF showed a blue-shift of emission (Figure 2e, f \& j, Supplementary Table S4).

The difference in thermal stability and reassembly of Tm-Enc, $M x$-Enc and Qt-Enc points to kinetic complexity in the encapsulin assembly pathway. For $M x$-Enc, aggregation of the unfolded/disassembled state is likely the main obstacle to achieving proper reassembly. Our $\mathrm{GuHCl}$ data also suggest that the $M x$-Enc unfolded/disassembled state(s) is less stable than for Tm-Enc or Qt-Enc. Whilst Qt-Enc began Trp solvation at $40^{\circ} \mathrm{C}$, DLS showed it did not change in size until $80^{\circ} \mathrm{C}$, indicating $Q t$-Enc undergoes structural change from $40^{\circ} \mathrm{C}$, but remains assembled until $80^{\circ} \mathrm{C}$. The stability of Tm-Enc and $Q t-E n c$ at high temperatures are consistent with them being derived from thermophilic bacteria. ${ }^{6,12}$ Whereas $M x-E n c$ is derived from bacteria that lives in soil ${ }^{45}$, which may explain its lower tolerance to temperature. 


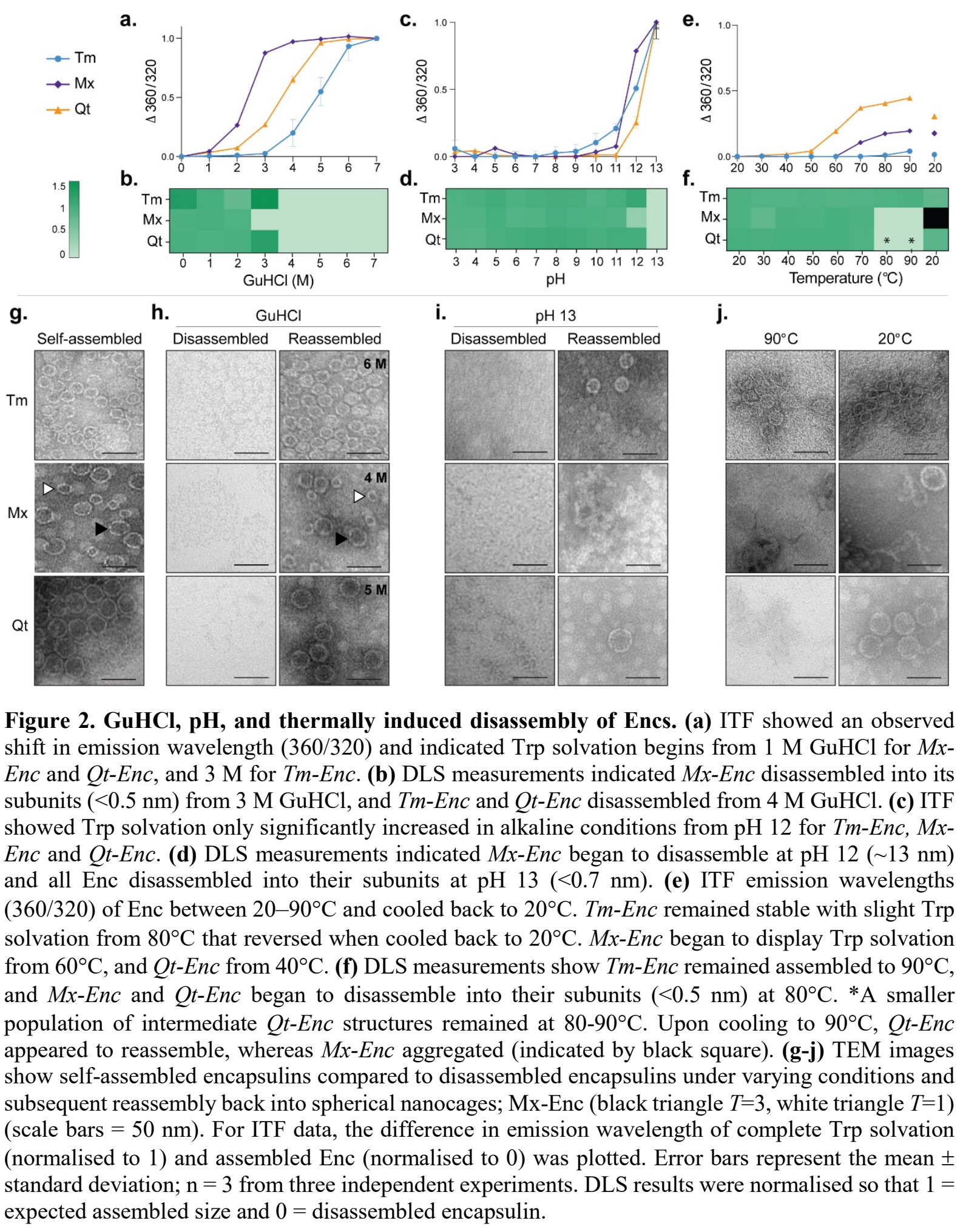

\section{Alternate disassembly conditions}

We have begun to use the ITF technique, developed herein, to investigate other chemical additives that may alter the folding and/or assembly of encapsulins, including redox conditions (10 mM DTT or $\mathrm{H}_{2} \mathrm{O}_{2}$ ) and ionic environments $(1 \mathrm{M} \mathrm{NaCl})$. However, we are yet to find 
another condition to induce disassembly. All Encs remained stable in $\mathrm{NaCl}$, and only $M x-E n c$ displayed a small blue-shift in emission in $\mathrm{H}_{2} \mathrm{O}_{2}$, and an observable increase in diameter after incubation in either DTT or $\mathrm{H}_{2} \mathrm{O}_{2}$ (Supplementary Figure S4, Supplementary Table S7). This may be due to reduction/oxidation of disulfide bonds causing a weaker association between subunits, resulting in swelling of the nanocage. ${ }^{46}$

\section{Encapsulin Reassembly}

Based on the above results, high concentrations $(6 \mathrm{M})$ of $\mathrm{GuHCl}$ and $\mathrm{pH} 13$ conditions were selected for further analysis of the Tm-Enc, $M x$-Enc, and Qt-Enc reassembly mechanism. Notably, these conditions disassemble encapsulin to differing degrees. ITF revealed maximum peak emissions for disassembly with $\mathrm{pH} 13$ to be $\sim 341 \mathrm{~nm}$ for Tm-Enc, and $\sim 347 \mathrm{~nm}$ for $M x$ Enc and Qt-Enc, whereas disassembly with $6 \mathrm{M} \mathrm{GuHCl}$ resulted in a maximum peak emission at $\sim 354 \mathrm{~nm}$ for all encapsulins (Figure 3a-c). As previously discussed, a Trp emission maxima $\geq 350 \mathrm{~nm}$ (as observed for all encapsulins in $6 \mathrm{M} \mathrm{GuHCl}$ ) is expected for unfolded protein. However, in $\mathrm{pH} 13$, all encapsulins display a Trp max emission $<350 \mathrm{~nm}$, suggesting that at least some of their subunits' secondary structure remains intact.

Reassembly of all encapsulins after disassembly in either $6 \mathrm{M} \mathrm{GuHCl}$ or $\mathrm{pH} 13$ was initiated

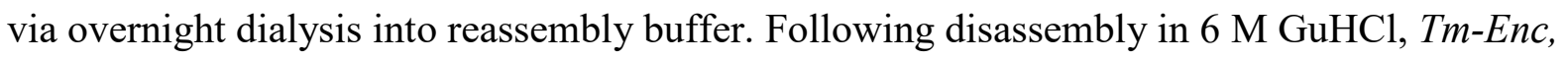
$M x-E n c$, and $Q t-E n c$ all reassembled back to their original structures, as indicated by ITF maximum emission and DLS size (Figure 3a-c, Supplementary Figure S3). In contrast, after disassembly in $\mathrm{pH} 13$, only Tm-Enc reassembled to its original structure. For $M x$-Enc and $Q t$ Enc, a blue-shift in ITF emission, resembling the Trp maximum emission of the original selfassembled material, was observed. However, native-PAGE results showed no bands (Supplementary Figure S3), and TEM analysis found no structures for $M x$-Enc, and only a single nanocage was imaged for Qt-Enc (Figure 2i). Indeed, DLS analysis of $\mathrm{pH} 13$ reassembled $M x$-Enc and $Q t$-Enc showed the presence of either large aggregates or smaller structures $(\sim 17 \mathrm{~nm})$, which suggests the subunits may be reassembling into smaller cages and/or intermediate structures at a concentration too low to detect by native-PAGE and TEM.

As previously discussed, at $\mathrm{pH} 13$, hydrolysis of the peptide bonds can occur, leading to protein misfolding and/or aggregation ${ }^{44}$, which may account for some of this inefficiency in encapsulin reassembly. Interestingly, although the position of the Trp emission peak for the original self-assembled, $\mathrm{GuHCl}$ reassembled and $\mathrm{pH} 13$ reassembled samples of Qt-Enc are not significantly different, a difference in spectral shape is noted for the $\mathrm{pH} 13$ reassembled sample. The blue-shifted shoulder of the Qt-Enc Trp emission peak, thought to correspond to 
Trp95 buried in the pentamer/hexamer interface of the intact nanocage, is lower in intensity for the $\mathrm{pH} 13$ reassembled sample. This result is consistent with reassembly of a different structure with a native-like fold, instead of the original intact $T=4 Q t$-Enc nanocage, upon reassembly from $\mathrm{pH}$ 13. The reassembly of $M x-E n c$ and $Q t-E n c$ after $\mathrm{pH} 13$ disassembly into smaller nanocages and/or alternative structures in inefficient quantities suggests this pathway, in its native form, may not be suitable for some applications.
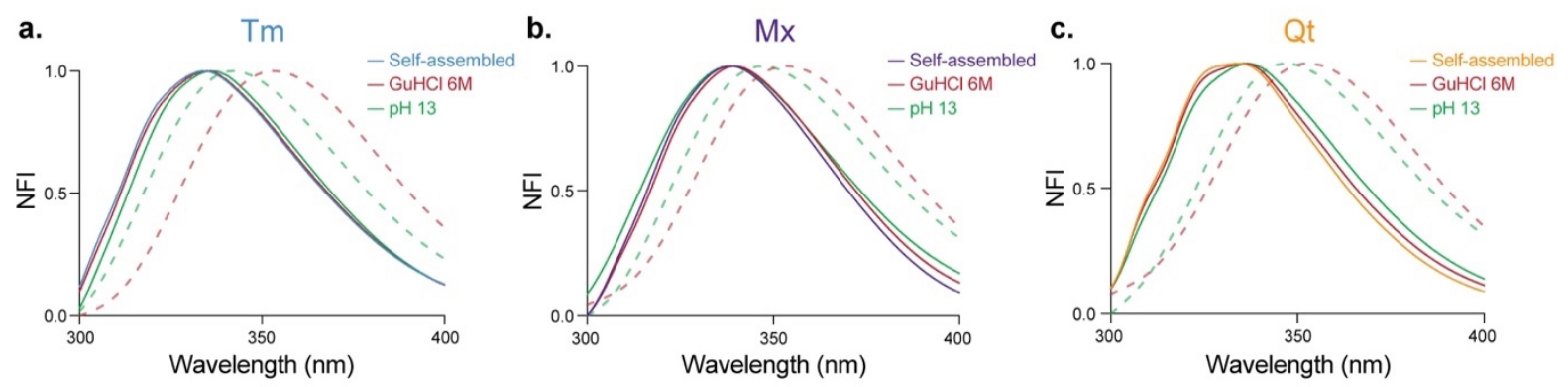

Figure 3. Comparison of Enc spectra of self-assembled, disassembled in GuHCl $6 \mathrm{M}$ or pH 13, and subsequent reassembly. (a) Tm-Enc ITF emission when self-assembled (blue), disassembled with $6 \mathrm{M} \mathrm{GuHCl}$ (dotted red), reassembled after $6 \mathrm{M} \mathrm{GuHCl}$ disassembly (solid red), disassembled with $\mathrm{pH}$ 13 (dotted green), and reassembled after $\mathrm{pH} 13$ disassembly (solid green). (b) $M x$-Enc ITF emission when self-assembled (purple), disassembled with $6 \mathrm{M} \mathrm{GuHCl}$ (dotted red), reassembled after $6 \mathrm{M}$ $\mathrm{GuHCl}$ disassembly (solid red), disassembled with $\mathrm{pH} 13$ (dotted green), and reassembled after $\mathrm{pH} 13$ disassembly (solid green). (c) $Q t$-Enc ITF emission when self-assembled (orange), disassembled with $6 \mathrm{M} \mathrm{GuHCl}$ (dotted red), reassembled after $6 \mathrm{M} \mathrm{GuHCl}$ disassembly (solid red), disassembled with $\mathrm{pH}$ 13 (dotted green), and reassembled after $\mathrm{pH} 13$ disassembly (solid green).

\section{Timescale of encapsulin reassembly}

To advance encapsulins as a cargo-carrying platform, disassembled encapsulin needs to be able to be reassembled on a viable timescale. The encapsulin nanocages were disassembled for $1 \mathrm{~h}$ via $6 \mathrm{M} \mathrm{GuHCl}$ or $\mathrm{pH} 13$ before reassembly was initiated using dialysis, with samples measured with ITF and DLS every 15 min over a 75 min timeframe. The rate of reassembly appeared to vary between each Enc. Tm-Enc reassembled faster than $M x$-Enc and Qt-Enc, with DLS indicating Tm-Enc reached its assembled diameter within 15 min after being disassembled via $\mathrm{pH} \mathrm{13}$, and between 15-30 min when disassembled via $6 \mathrm{M} \mathrm{GuHCl}$ (Figure $4 \mathrm{a} \& \mathrm{~b}$, Supplementary Table S5 \& S6). The faster reassembly after pH 13 disassembly compared to $\mathrm{GuHCl}$ may be explained by the subunits still maintaining some structure after incubation in pH 13 conditions. However, Tm-Enc reassembly after pH 13 disassembly displayed a more gradual Trp burial and a slight decrease in size at 45 mins (Figure 4a \& b, Supplementary Table S6). This suggests reassembly after $\mathrm{pH} 13$ may follow a more dynamic pathway, with rapid nanocage formation, followed by restructuring. In contrast, after $6 \mathrm{M} \mathrm{GuHCl}$ disassembly, $Q t$ Enc reassembled into its original diameter between 30-45 min, and $M x$-Enc appeared to 
partially assemble between 45-60 min, but only completely assembled between 60-75 min (Figure $4 \mathrm{a} \& \mathrm{~b}$, Supplementary Table S5). The difference in rates between encapsulins may reflect the overall stability of the complex i.e., complexes that are more stable may reassemble quicker. In a recent report, the in vivo loading of cargo proteins into Tm-Enc during its selfassembly was found to be $\sim 8$ times less efficient than with $M x-E n c .{ }^{47}$ The authors suggested that this striking difference was partly due to the smaller Tm-Enc self-assembling at a faster rate than $M x-E n c$, thus limiting the available contact time between ESig-tagged cargo and the nanocage's interior surface. This hypothesis is now supported by our observation that Tm-Enc reassembles up to four times quicker than the larger $M x$-Enc (Figure 4). This therefore highlights the critical role assembly time-frames play in cargo-loading efficiency, and that modifying such properties can enhance cargo packing, density and/or stoichiometry.

The rapid burial of Trp residues for all encapsulins within $15 \mathrm{~min}$, as shown by ITF, prior to formation of the cages, suggests a reassembly pathway where subunits first fold into an intermediate structure before gradually forming the cage. A previous study on encapsulin from $B$. linens $(T=1)$ suggested reassembly occurred with subunits first forming stable dimers prior to the final nanocage formation, with a preference for even numbered stoichiometries as demonstrated via mass spectrometry analysis. ${ }^{38}$ Additionally, ferritin has been found to have a biphasic reassembly, where an initial fast step occurs with folding of subunits and unknown stable intermediates, followed by the slower restructuring of intermediates into the nanocage. ${ }^{23}$ Data of $\mathrm{pH} 13$ reassembly rate for $M x-E n c$ and Qt-Enc was not included, as the presence of aggregation prevented extraction of accurate DLS size values. This suggests that reassembly from the $\mathrm{pH} 13$ disassembled state for the larger and more complex Mx-Enc and Qt-Enc may be prone to protein misfolding pathways and/or partial assembly, and thus, without additional re-engineering, may not be ideal for use in future applications. 

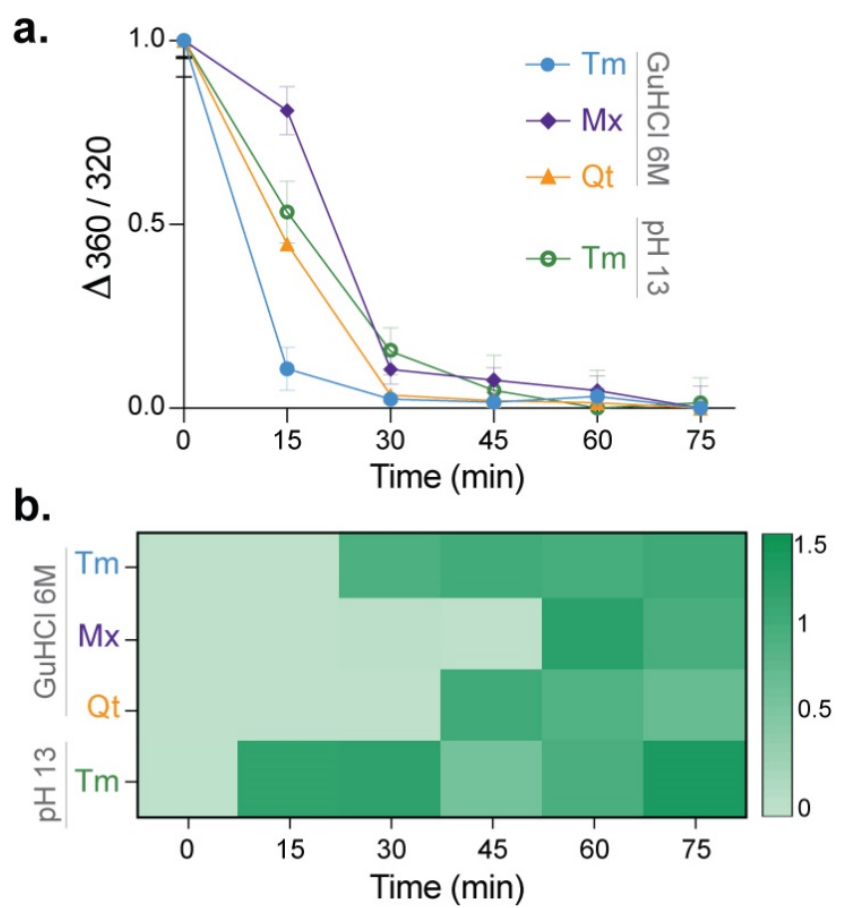

Figure 4. Reassembly timeframes of encapsulins. (a) ITF shift in emission wavelength $(\Delta 360 / 320)$ of Tm-Enc, $M x$-Enc, and Qt-Enc disassembled via $6 \mathrm{M} \mathrm{GuHCl}$ and $T m$-Enc disassembled via $\mathrm{pH} 13$ and reassembly measured every $15 \mathrm{~min}$. The difference in emission wavelength of disassembled Enc (normalised to 1) and assembled Enc at $75 \mathrm{~min}$ (normalised to 0) was plotted. Error bars represent the mean \pm standard deviation; $\mathrm{n}=3$ from three independent experiments. (b) Heatmap of DLS measurements of $T m-E n c, M x$-Enc, and $Q t$-Enc disassembled via $6 \mathrm{M} \mathrm{GuHCl}$ and $T m$-Enc disassembled via $\mathrm{pH} 13$ and reassembly measured every $15 \mathrm{~min}$. DLS results were normalised so that $1=$ expected assembled size and $0=$ disassembled encapsulin.

\section{Nanomechanical stability of self-assembled vs reassembled encapsulins}

To understand any changes in the structural integrity of Tm-Enc, Mx-Enc, and Qt-Enc after reassembly in the conditions tested above, AFM was utilised to compare the morphology, rupture point, and elasticity between self-assembled and reassembled encapsulins. Previous AFM analysis has been done on the rigidity of Tm-Enc and encapsulin from B. linens $(T=1)$, and suggested that the presence of cargo within encapsulin from $B$. linens may lead to some destabilisation, as indicated by a lower rupture force in loaded versus unloaded encapsulin. ${ }^{38}$ However, the effect of reassembly on the nanomechanical stability on $T m$-Enc, as well as the larger and more structurally complex $M x-E n c$ and $Q t-E n c$, is yet to be elucidated.

Individual encapsulins were imaged with a scan size of $\sim 100 \mathrm{~nm} \times 100 \mathrm{~nm}$ to visualise their topographical detail. Self-assembled encapsulins were found to be at their expected size, including both $T=3$ and $T=1$ conformations of $M x$-Enc (Figure 5a). However, clear morphological variation can be seen after reassembly, with all encapsulins demonstrating flattening of their surfaces. Additionally, $Q t-E n c$ appeared more aggregated after reassembly. 
Being composed of similar constituents, approximately the same elasticity would be expected between each self-assembled encapsulin, which was found to be the case of Tm-Enc and $M x$-Enc, with an average elasticity of $2.77 \pm 0.96$ and $2.12 \pm 0.81 \mathrm{MPa}$, respectively (Figure 5b, Supplementary Figure S5). However, Qt-Enc displayed a higher average elasticity of $25.60 \pm 14.92 \mathrm{MPa}$, indicating it has greater structural integrity and is therefore more resilient to deformation (Figure 5b, Supplementary Figure S5). Qt-Enc may therefore be more strongly self-assembled than $T m-E n c$ and $M x-E n c$, which could be attributed to its higher structural complexity, as the different symmetries and subunit-subunit contacts may influence the strength of interfacial interactions. ${ }^{48}$ Upon reassembly following $6 \mathrm{M} \mathrm{GuHCl}$ disassembly, the elasticity of all encapsulins decreased significantly, with $T m$-Enc average elasticity $1.16 \pm$ $0.93 \mathrm{MPa}(58.12 \%$ decrease), Mx-Enc $1.39 \pm 0.78 \mathrm{MPa}(34.43 \%$ decrease), and Qt-Enc $0.85 \pm$ $0.62 \mathrm{MPa}(96.68 \%$ decrease), indicating a significant reduction in structural integrity (Figure 5b, Supplementary Figure S5). Elasticity data of reassembled Tm-Enc after disassembly via $\mathrm{pH}$ 13 was not included, as, during testing, the sample became unstructured and did not retain any elasticity or show any elastic response. This suggests that even though Tm-Enc reassembled after disassembly via $\mathrm{pH} 13$, these conditions may have irreversibly altered the protein by hydrolysis, thereby affecting stability. ${ }^{44}$

The rupture force represents the maximum force a protein cage withstands prior to puncture by the AFM tip. ${ }^{49}$ The rupture force of all self-assembled encapsulins were between $\sim 0.1-0.2$ $\mathrm{nN}$, indicating they are fairly fragile (Figure $5 \mathrm{~b}$ ). This is in contrast to AFM results from a previous study on self-assembled empty Tm-Enc and encapsulin from B. linens, which had rupture forces of 0.63 and $0.64 \mathrm{nN}$, respectively ${ }^{38}$, indicating that the encapsulins used in the current study are slightly more fragile. After reassembly, the rupture force decreased further by a factor of $\sim 5$ for each encapsulin system tested (Figure $5 \mathrm{~b}$ ).

Together, these results show that, after undergoing disassembly in either $6 \mathrm{M} \mathrm{GuHCl}$ or $\mathrm{pH}$ 13 and subsequent reassembly, all three encapsulin architectures significantly lose structural integrity. In terms of encapsulins loaded with cargo under in vitro conditions, the observed increased fragility could be beneficial if wanting a system that can be easily broken down to release cargo, such as intracellular drug delivery or immunotherapy. If in need of a more robust system (e.g., enzyme-loaded nanocages for industrial biocatalysis), however, these results demonstrate the importance to understand and modify encapsulins so they can disassemble in more benign conditions. Furthermore, to enhance understanding of the reassembly pathway of encapsulins, future studies could assess real-time assembly of the subunits. This has been 
achieved with large bacterial microcompartments, where AFM revealed that the shell facets assemble from preformed oligomers. ${ }^{50}$
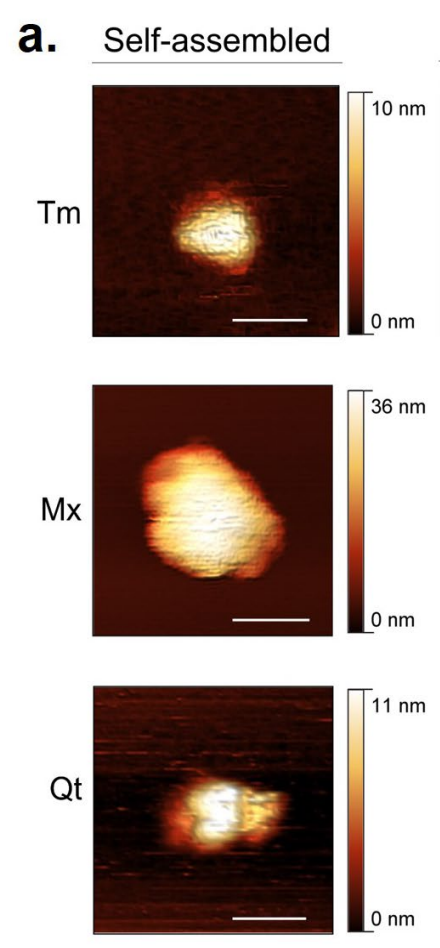

Reassembled
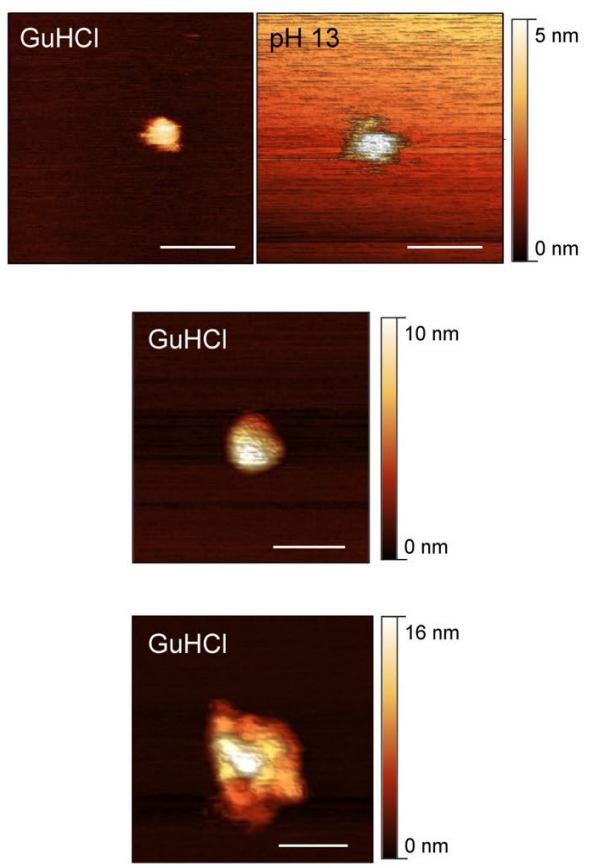

b.
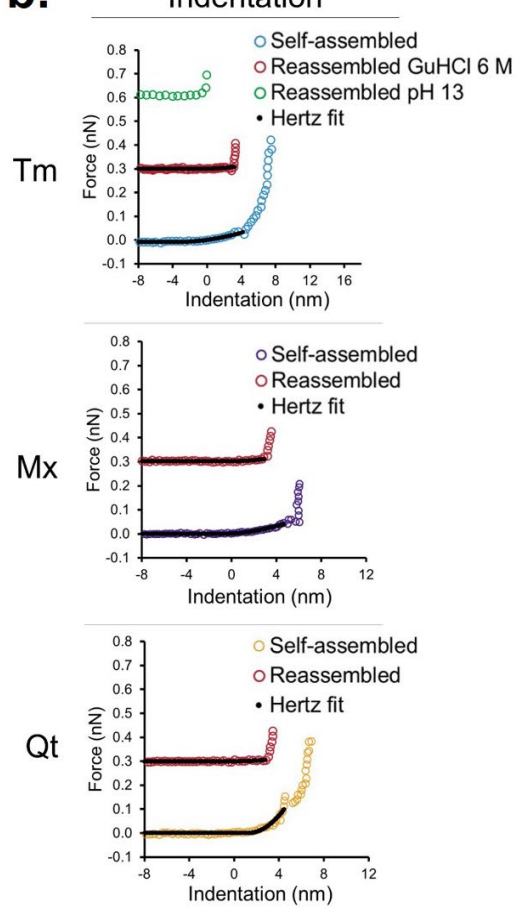

Figure 5. AFM imaging and elasticity comparing self-assembled/reassembled encapsulins. (a) Nanoscale AFM images obtained for individual encapsulins comparing morphologies of self-assembled $T m$-Enc, $M x$-Enc, and Qt-Enc with encapsulins disassembled via $\mathrm{pH} 13$ or $\mathrm{GuHCl}(6 \mathrm{M})$ and subsequently reassembled. White scale bars represent $30 \mathrm{~nm}$. (b) Representative indentation curves and Hertz Fit of encapsulins used to determine Young's elastic modulus (E) using Hertz equation to convert experimentally derived force versus distance curves into indentation data (coloured circles) and to fit the elastic (non-linear) region to calculated $\mathrm{E}$ values (black lines).

\section{Conclusion}

In summary, this study characterised the conditions facilitating the disassembly and reassembly of $T m-E n c, M x-E n c$, and $Q t-E n c$, with each encapsulin system varying in degree of sensitivity to $\mathrm{GuHCl}, \mathrm{pH}$, and temperature. When disassembled in high $\mathrm{pH}$ conditions, the subunits appeared to individually remain structurally intact, as opposed to the complete unravelling observed when disassembled in high concentrations of GuHCl. Furthermore, Tm$E n c, M x$-Enc, and $Q t$-Enc were all able to reassemble within 75 min after disassembly with $\mathrm{GuHCl}$; however, only Tm-Enc was able to reassemble after disassembly at $\mathrm{pH} 13$ (within 15 min). Our results suggest that disassembly by $\mathrm{GuHCl}$ and $\mathrm{pH} 13$ affect different interfacial interactions. Self-assembly, disassembly, and reassembly of protein nanocages are highly dependent on interfacial subunit interaction ${ }^{17}$, and therefore, further understanding of these 
interactions is a fundamental step required to harness their potential. Furthermore, using AFM, we revealed a previously unknown effect of in vitro disassembly/reassembly on encapsulin stability, finding that encapsulin loses a substantial amount of structural integrity when disassembled and subsequently reassembled. These findings have implications if requiring in vitro loaded encapsulin for a particular application, but may be beneficial when nanocage dissociation and cargo release is favored.

In high concentrations of $\mathrm{GuHCl}$, all encapsulins completely unfold and refold efficiently; however, these harsh conditions pose limitations for applications involving in vitro loading of cargo that may be sensitive to these conditions, as it could be destroyed in the process. Furthermore, the need to use high concentrations of $\mathrm{GuHCl}$ or alkaline $\mathrm{pH}$ in order to achieve disassembly may limit use for biomedical applications, where biocompatibility is paramount. Therefore, understanding the disassembly/reassembly conditions is an important step to elucidate target regions within encapsulin subunits that can be engineered to allow nanocage disassembly to occur under milder conditions. For example, the elucidation of ferritin disassembly/reassembly conditions has allowed for targeted amino acid engineering to enhance its potential as a nanocarrier for $\mathrm{pH}$-sensitive compounds. As previously discussed, ferritin requires strong acidic conditions $(\mathrm{pH} \leq 2)$ to disassemble, and molecules sensitive to these conditions are unable to be loaded. Therefore, by removing the last 23 amino acids from the carboxyl terminal, researchers found that ferritin was able to disassemble at $\mathrm{pH} 4$ and reassemble again at $\mathrm{pH} 7$, with the ability to load the bioactive agent curcumin and maintain its regular structure. ${ }^{24}$

Engineering interfaces can also allow enhanced control over size and cargo-loading efficiency of protein nanocages. For instance, due to being disassembled under high acidic conditions, a lot of ferritin is lost during this process and therefore it has a low loading efficiency. Consequently, ferritin variant subunit interfaces were engineered to display His6 motifs, which allowed nanocages to reassemble in the presence of either transition metal ions or $\mathrm{pH} 10$, which increased loading efficiency by 1.6-3.6 times compared with previous acid disassembly. ${ }^{51}$ Due to ferritin being limited to one size, researchers engineered the subunit interface by first identifying the key subunit interfaces and removing the amino acids that do not participate in the interfacial interactions, resulting in the formation of a $17 \mathrm{~nm} 48$-er nanocage instead of the $12 \mathrm{~nm}$ 24-er native ferritin structure. ${ }^{52}$ With an increased understanding of encapsulin structure, disassembly/reassembly conditions, molecular mechanisms, stability, and stoichiometry of intermediate structures, future genetic engineering efforts of subunits may 
similarly lead to enhanced control over conditions that trigger disassembly/reassembly, size, structure, and cargo-loading efficiency of encapsulins.

In summary, the findings of this study advance our understanding of encapsulins by providing critical insight into their unique disassembly/reassembly dynamics. This knowledge provides a roadmap towards an encapsulin "tool-kit" comprised of nanocages with varying structural architectures and biochemical/biophysical properties, that can be readily selected and further customised for a specific nanobiotechnological application.

\section{ASSOCIATED CONTENT}

\section{Supporting Information.}

List of genetic constructs, examples of purification chromatographs, gel images of purified encapsulins, molecular graphics of Trp residue positions within encapsulin subunits, gel images of disassembled/reassembled encapsulins, stability study of encapsulins in REDOX and ionic condition, AFM elasticity histograms, and DLS measurement tables.

\section{AUTHOR INFORMATION}

Corresponding Author

Andrew Care

Email: andrew.care@uts.edu.au

\section{Author Contributions}

I.B co-designed the research, generated all nanocage constructs, conducted all disassembly/reassembly characterisation work, performed data analysis and wrote the manuscript. S.G assisted in experimental design, data interpretation, and wrote the manuscript. D.D assisted in protein production and purification, TEM imaging, protein modelling, and revised the manuscript. A.E performed AFM experiments and data analysis. L.C.P supervised the project and revised the manuscript. A.C. conceptualised and co-designed the study, supervised the project, and wrote the manuscript.

\section{Notes}

The authors declare no competing financial interest. 
bioRxiv preprint doi: https://doi.org/10.1101/2021.06.06.447285; this version posted June 8,2021 . The copyright holder for this preprint

(which was not certified by peer review) is the author/funder, who has granted bioRxiv a license to display the preprint in perpetuity. It is made available under aCC-BY-NC 4.0 International license.

\section{ACKNOWLEDGMENTS}

I.B. is supported by a Dementia Australia Research Foundation PhD scholarship. A.C. is supported by the UTS Chancellor's Postdoctoral Research Fellowship scheme. This work was supported by grants to A.C and L.C.P by the Dementia Australia Research Foundation, the National Foundation for Medical Research and Innovation, the Brain Foundation and the NeuroSurgical Research Foundation.

\section{References}

1. Diaz, D.; Care, A.; Sunna, A. J. G., Bioengineering Strategies for Protein-Based Nanoparticles. 2018, 9, (7), 370.

2. $\quad$ Sandra, F.; Khaliq, N. U.; Sunna, A.; Care, A., Developing protein-based nanoparticles as versatile delivery systems for cancer therapy and imaging. Nanomaterials 2019, 9, (9), 1329. 3. Jones, J. A.; Giessen, T. W., Advances in encapsulin nanocompartment biology and engineering. Biotechnology and bioengineering 2021, 118, (1), 491-505.

4. Gabashvili, A. N.; Chmelyuk, N. S.; Efremova, M. V.; Malinovskaya, J. A.; Semkina, A. S.; Abakumov, M. A., Encapsulins-Bacterial protein nanocompartments: Structure, properties, and application. Biomolecules 2020, 10, (6), 966.

5. $\quad$ Sutter, M.; Boehringer, D.; Gutmann, S.; Gunther, S.; Prangishvili, D.; Loessner, M. J.; Stetter, K. O.; Weber-Ban, E.; Ban, N., Structural basis of enzyme encapsulation into a bacterial nanocompartment. Nature structural \& molecular biology 2008, 15, (9), 939-47.

6. Giessen, T. W.; Orlando, B. J.; Verdegaal, A. A.; Chambers, M. G.; Gardener, J.; Bell, D. C.; Birrane, G.; Liao, M.; Silver, P. A., Large protein organelles form a new iron sequestration system with high storage capacity. Elife 2019, 8.

7. Sigmund, F.; Pettinger, S.; Kube, M.; Schneider, F.; Schifferer, M.; Aichler, M.; Schneider, S.; Walch, A.; Misgeld, T.; Dietz, H., Iron-sequestering nanocompartments as multiplexed Electron Microscopy gene reporters. bioRxiv 2019, 516955.

8. McHugh, C. A.; Fontana, J.; Nemecek, D.; Cheng, N.; Aksyuk, A. A.; Heymann, J. B.; Winkler, D. C.; Lam, A. S.; Wall, J. S.; Steven, A. C., A virus capsid-like nanocompartment that stores iron and protects bacteria from oxidative stress. The EMBO journal 2014, 33, (17), 1896-1911.

9. Giessen, T. W.; Silver, P. A., Widespread distribution of encapsulin nanocompartments reveals functional diversity. Nature microbiology 2017, 2, (6), 17029.

10. Contreras, H.; Joens, M. S.; McMath, L. M.; Le, V. P.; Tullius, M. V.; Kimmey, J. M.; Bionghi, N.; Horwitz, M. A.; Fitzpatrick, J. A.; Goulding, C. W., Characterization of a Mycobacterium tuberculosis nanocompartment and its potential cargo proteins. Journal of Biological Chemistry 2014, 289, (26), 18279-18289.

11. He, D.; Hughes, S.; Vanden-Hehir, S.; Georgiev, A.; Altenbach, K.; Tarrant, E.; Mackay, C. L.; Waldron, K. J.; Clarke, D. J.; Marles-Wright, J., Structural characterization of encapsulated ferritin provides insight into iron storage in bacterial nanocompartments. Elife 2016, 5, e18972.

12. Sutter, M.; Boehringer, D.; Gutmann, S.; Günther, S.; Prangishvili, D.; Loessner, M. J.; Stetter, K. O.; Weber-Ban, E.; Ban, N., Structural basis of enzyme encapsulation into a bacterial nanocompartment. Nature structural and molecular biology 2008, 15, (9), 939. 
13. Nichols, R. J.; LaFrance, B.; Phillips, N. R.; Radford, D. R.; Oltrogge, L. M.; ValentinAlvarado, L. E.; Bischoff, A. J.; Nogales, E.; Savage, D. F., Discovery and characterization of a novel family of prokaryotic nanocompartments involved in sulfur metabolism. Elife 2021, 10, e59288.

14. Cassidy-Amstutz, C.; Oltrogge, L.; Going, C. C.; Lee, A.; Teng, P.; Quintanilla, D.; East-Seletsky, A.; Williams, E. R.; Savage, D. F., Identification of a minimal peptide tag for in vivo and in vitro loading of encapsulin. Biochemistry 2016, 55, (24), 3461-3468.

15. Rahmanpour, R.; Bugg, T. D., Assembly in vitro of Rhodococcus jostii RHA 1 encapsulin and peroxidase DypB to form a nanocompartment. The FEBS journal 2013, 280, (9), 2097-2104.

16. Künzle, M.; Mangler, J.; Lach, M.; Beck, T., Peptide-directed encapsulation of inorganic nanoparticles into protein containers. Nanoscale 2018, 10, (48), 22917-22926.

17. McManus, J. J.; Charbonneau, P.; Zaccarelli, E.; Asherie, N., The physics of protein self-assembly. Current opinion in colloid \& interface science 2016, 22, 73-79.

18. Zhang, Y.; Orner, B. P., Self-assembly in the ferritin nano-cage protein superfamily. International journal of molecular sciences 2011, 12, (8), 5406-21.

19. Gerl, M.; Jaenicke, R., Mechanism of the self-assembly of apoferritin from horse spleen. European Biophysics Journal 1987, 15, (2), 103-109.

20. Gerl, M.; Jaenicke, R.; Smith, J. M.; Harrison, P. M., Self-assembly of apoferritin from horse spleen after reversible chemical modification with 2, 3-dimethylmaleic anhydride. Biochemistry 1988, 27, (11), 4089-4096.

21. Crichton, R. R.; Bryce, C. F., Subunit interactions in horse spleen apoferritin. Dissociation by extremes of pH. Biochemical Journal 1973, 133, (2), 289-299.

22. Kim, M.; Rho, Y.; Jin, K. S.; Ahn, B.; Jung, S.; Kim, H.; Ree, M., pH-dependent structures of ferritin and apoferritin in solution: disassembly and reassembly. Biomacromolecules 2011, 12, (5), 1629-1640.

23. Mohanty, A.; Jena, S. S.; Behera, R. K., Kinetics of Ferritin Self-Assembly by Laser Light Scattering: Impact of Subunit Concentration, $\mathrm{pH}$, and Ionic Strength. Biomacromolecules 2021.

24. Chen, H.; Zhang, S.; Xu, C.; Zhao, G., Engineering protein interfaces yields ferritin disassembly and reassembly under benign experimental conditions. Chemical Communications 2016, 52, (46), 7402-7405.

25. Diaz, D.; Care, A.; Sunna, A., Bioengineering Strategies for Protein-Based Nanoparticles. Genes (Basel) 2018, 9, (7), 370.

26. Collett, S.; Torresi, J.; Earnest-Silveira, L.; Christiansen, D.; Elbourne, A.; Ramsland, P. A., Probing and pressing surfaces of hepatitis C virus-like particles. Journal of colloid and interface science 2019, 545, 259-268.

27. Collett, S.; Torresi, J.; Silveira, L. E.; Truong, V. K.; Christiansen, D.; Tran, B. M.; Vincan, E.; Ramsland, P. A.; Elbourne, A., Investigating virus-host cell interactions: Comparative binding forces between hepatitis $\mathrm{C}$ virus-like particles and host cell receptors in 2D and 3D cell culture models. Journal of Colloid and Interface Science 2021, 592, 371-384. 28. Nečas, D.; Klapetek, P., Gwyddion: an open-source software for SPM data analysis. Open Physics 2012, 10, (1), 181-188.

29. Touhami, A.; Nysten, B.; Dufrêne, Y. F., Nanoscale Mapping of the Elasticity of Microbial Cells by Atomic Force Microscopy. Langmuir 2003, 19, (11), 4539-4543.

30. Lakowicz, J. R., Principles of fluorescence spectroscopy. 3rd ed. ed.; Springer: New York, 2006.

31. Sonotaki, S.; Noguchi, K.; Yohda, M.; Murakami, Y., A zeolite as a tool for successful refolding of PEGylated proteins and their reassembly with tertiary structures. Biotechnology progress 2019, 35, (5), e2853. 
32. Kelly, S. M.; Jess, T. J.; Price, N. C., How to study proteins by circular dichroism. Biochimica et Biophysica Acta (BBA)-Proteins and Proteomics 2005, 1751, (2), 119-139.

33. Bhattacharjee, C.; Das, K., Thermal unfolding and refolding of $\beta$-lactoglobulin: An intrinsic and extrinsic fluorescence study. European Journal of Biochemistry 2000, 267, (13), 3957-3964.

34. Reshetnyak, Y. K.; Burstein, E. A., Decomposition of protein tryptophan fluorescence spectra into log-normal components. II. The statistical proof of discreteness of tryptophan classes in proteins. Biophysical journal 2001, 81, (3), 1710-1734.

35. Chaudhary, A. P.; Vispute, N. H.; Shukla, V. K.; Ahmad, B., A comparative study of fibrillation kinetics of two homologous proteins under identical solution condition. Biochimie 2017, 132, 75-84.

36. Pettersen, E. F.; Goddard, T. D.; Huang, C. C.; Meng, E. C.; Couch, G. S.; Croll, T. I.; Morris, J. H.; Ferrin, T. E., UCSF ChimeraX: Structure visualization for researchers, educators, and developers. Protein Sci 2021, 30, (1), 70-82.

37. Zhou, B.; Wan, J.; Wang, J.; Cao, X., Effect of chaotropes in reverse micellar extraction of kallikrein. Process Biochemistry 2012, 47, (2), 229-233.

38. Snijder, J.; Kononova, O.; Barbu, I. M.; Uetrecht, C.; Rurup, W. F.; Burnley, R. J.; Koay, M. S.; Cornelissen, J. J.; Roos, W. H.; Barsegov, V., Assembly and mechanical properties of the cargo-free and cargo-loaded bacterial nanocompartment encapsulin. Biomacromolecules 2016, 17, (8), 2522-2529.

39. Martsev, S. P.; Vlasov, A. P.; Arosio, P., Distinct stability of recombinant L and H subunits of human ferritin: calorimetric and ANS binding studies. Protein engineering 1998, $11,(5), 377-381$.

40. Liang, M.; Fan, K.; Zhou, M.; Duan, D.; Zheng, J.; Yang, D.; Feng, J.; Yan, X., Hferritin-nanocaged doxorubicin nanoparticles specifically target and kill tumors with a singledose injection. Proceedings of the National Academy of Sciences 2014, 111, (41), 1490014905.

41. Yang, R.; Liu, Y.; Blanchard, C.; Zhou, Z., Channel directed rutin nano-encapsulation in phytoferritin induced by guanidine hydrochloride. Food chemistry 2018, 240, 935-939.

42. Sharma, J.; Uchida, M.; Miettinen, H. M.; Douglas, T., Modular interior loading and exterior decoration of a virus-like particle. Nanoscale 2017, 9, (29), 10420-10430.

43. Gasteiger, E.; Gattiker, A.; Hoogland, C.; Ivanyi, I.; Appel, R. D.; Bairoch, A., ExPASy: the proteomics server for in-depth protein knowledge and analysis. Nucleic acids research 2003, 31, (13), 3784-3788.

44. Zhou, H.-X.; Pang, X., Electrostatic interactions in protein structure, folding, binding, and condensation. Chemical reviews 2018, 118, (4), 1691-1741.

45. Vos, M.; Velicer, G. J., Genetic population structure of the soil bacterium Myxococcus xanthus at the centimeter scale. Applied and environmental microbiology 2006, 72, (5), 36153625.

46. Asor, R.; Khaykelson, D.; Ben-nun-Shaul, O.; Oppenheim, A.; Raviv, U., Effect of calcium ions and disulfide bonds on swelling of virus particles. ACS omega 2019, 4, (1), 5864.

47. Altenburg, W. J.; Rollins, N.; Silver, P. A.; Giessen, T. W., Exploring targeting peptideshell interactions in encapsulin nanocompartments. Scientific reports 2021, 11, (1), 1-9.

48. Heinze, K.; Sasaki, E.; King, N. P.; Baker, D.; Hilvert, D.; Wuite, G. J.; Roos, W. H., Protein nanocontainers from nonviral origin: testing the mechanics of artificial and natural protein cages by AFM. The Journal of Physical Chemistry B 2016, 120, (26), 5945-5952.

49. de Pablo, P. J., The application of atomic force microscopy for viruses and protein shells: imaging and spectroscopy. Advances in virus research 2019, 105, 161-187. 
50. $\quad$ Sutter, M.; Faulkner, M.; Aussignargues, C.; Paasch, B. C.; Barrett, S.; Kerfeld, C. A.; Liu, L.-N., Visualization of bacterial microcompartment facet assembly using high-speed atomic force microscopy. Nano letters 2016, 16, (3), 1590-1595.

51. Gu, C.; Zhang, T.; Lv, C.; Liu, Y.; Wang, Y.; Zhao, G., His-Mediated Reversible SelfAssembly of Ferritin Nanocages through Two Different Switches for Encapsulation of Cargo Molecules. ACS nano 2020, 14, (12), 17080-17090.

52. Zhang, S.; Zang, J.; Zhang, X.; Chen, H.; Mikami, B.; Zhao, G., "Silent" amino acid residues at key subunit interfaces regulate the geometry of protein nanocages. ACS nano 2016, 10, (11), 10382-10388. 